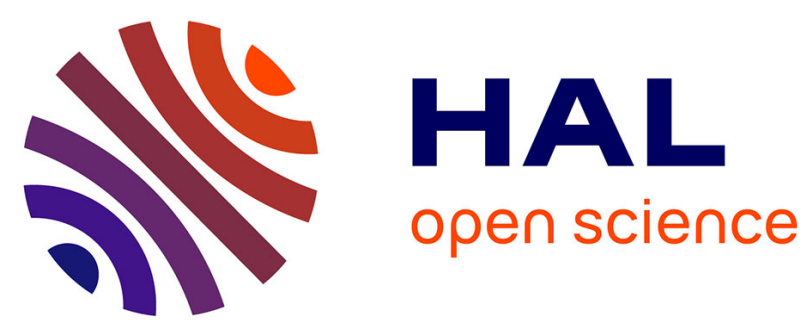

\title{
Real-Life Benefits of Statins for Cardiovascular Prevention in Elderly Subjects: A Population-Based Cohort Study
}

Julien Bezin, Nicholas Moore, Yohann Mansiaux, P. G. Steg, Antoine Pariente

\section{- To cite this version:}

Julien Bezin, Nicholas Moore, Yohann Mansiaux, P. G. Steg, Antoine Pariente. Real-Life Benefits of Statins for Cardiovascular Prevention in Elderly Subjects: A Population-Based Cohort Study. The American Journal of Medicine, 2019, 132 (6), pp.740-748 e7. 10.1016/j.amjmed.2018.12.032 . hal03211447

\section{HAL Id: hal-03211447 \\ https://hal.science/hal-03211447}

Submitted on 25 Oct 2021

HAL is a multi-disciplinary open access archive for the deposit and dissemination of scientific research documents, whether they are published or not. The documents may come from teaching and research institutions in France or abroad, or from public or private research centers.
L'archive ouverte pluridisciplinaire HAL, est destinée au dépôt et à la diffusion de documents scientifiques de niveau recherche, publiés ou non, émanant des établissements d'enseignement et de recherche français ou étrangers, des laboratoires publics ou privés.

\section{다)(1) $(5$}

Distributed under a Creative Commons Attribution - NonCommerciall 4.0 International 


\section{Real-life benefits of statins for cardiovascular prevention in elderly subjects: a population-based cohort study}

Julien Bezin, PharmD, $\mathrm{PhD}^{1}$, Nicholas Moore, $\mathrm{MD}, \mathrm{PhD}^{1,2,3}$, Yohann Mansiaux, $\mathrm{PhD}^{1}$, Philippe Gabriel Steg, MD, $\mathrm{PhD}^{4,5}$, Antoine Pariente, $\mathrm{MD}, \mathrm{PhD}^{1,2}$

${ }^{1}$ Univ. Bordeaux, INSERM, Bordeaux Population Health Research Center, team pharmacoepidemiology, UMR 1219, Bordeaux, France

${ }^{2} \mathrm{CHU}$ de Bordeaux, Bordeaux, France

${ }^{3}$ Bordeaux PharmacoEpi, INSERM CIC1401, Bordeaux, France

${ }^{4}$ FACT (French Alliance for Cardiovascular Clinical Trials), DHU FIRE, University Paris Diderot, AP-HP, INSERM U-1148, Paris, France

${ }^{5}$ NHLI, ICMS, Royal Brompton Hospital, Imperial College, London, United Kingdom

Corresponding author: Julien Bezin, Pharmacoepidemiology and Population Impact of Drugs, Bordeaux Population Health Research Center, University of Bordeaux, Case 121, 146 rue Léo Saignat, F-33076 Bordeaux cedex, France, Phone: +33 557571560, julien.bezin@ubordeaux.fr

Word count: 3313

Article type: Clinical Research Study

Funding source(s): None

Conflict of interest: All authors have completed and submitted the ICMJE Form for Disclosure of Potential Conflicts of Interest and declare: no support from any organization for 
the submitted work; PGS reports speaking or consulting fees from Amarin, Amgen, AstraZeneca, Bayer/Janssen, Boehringer-Ingelheim, Bristol-Myers-Squibb, Lilly, Merck, Novartis, Pfizer, Regeneron, Sanofi, Servier, and research grants from Bayer, Merck, Sanofi, and Servier; NM is chief executive officer of the Bordeaux PharmacoEpi platform that performs post-authorization studies (none related to the evaluation of statins) financed by pharmaceutical industries including those marketing or having marketed statins; grants of the French Medicines Agency (JB, YM, NM, and AP) and collaborations with public regulatory agencies and with health care insurance systems (NM, and AP) that might have an interest in the submitted work.

Authorship: Dr Bezin had full access to all of the data in the study and takes responsibility for the integrity of the data and the accuracy of the data analysis.

Study concept and design: JB, NM, YM, AP.

Acquisition, analysis, or interpretation of data: JB, NM, YM, PGS, AP.

Drafting of the manuscript: JB, YM.

Critical revision of the manuscript for important intellectual content: JB, NM, YM, PGS, AP. Statistical analysis: JB, YM.

Study supervision: AP.

Keywords: Statin; Cardiovascular risk; Comparative Effectiveness Research; Pharmacoepidemiology; Population-wide database

Running head: Statins for cardiovascular prevention in elderly 


\section{Abstract}

Objectives: The benefits of initiating statins in the elderly remains debated. We evaluated the effects of initiating statins in the elderly, according to cardiovascular risk.

Methods: This population-based cohort study used data of the representative sample of the French healthcare system database for the 2008-2015 period. New users of statins, aged 75 years and above were dynamically included in the cohort and matched 1:1 to statin non-users on age, gender, numbers of different drugs dispensed and medical consultations, and cardiovascular history. Patients were classified in three cardiovascular risk groups: secondary prevention (history of coronary heart disease), primary prevention with modifiable risk factors (diabetes or cardiovascular medications), and primary prevention without modifiable risk factor (none of the above). Effect of cumulative use of statins on occurrence of acute coronary syndrome or all-cause death was analyzed by using multivariable time-dependent Cox models stratified on cardiovascular risk at inclusion.

Results: Among the 7284 patients included, median follow-up was 4.7 years. Cumulative use of statins was associated with a lower risk of outcomes in the primary prevention with modifiable risk factors group (adjusted hazard ratio 0.93 per year of use; $95 \%$ confidence interval $0.89-0.96 ; \mathrm{p}<0.01)$, and in the secondary prevention group $(0.75 ; 0.63-0.90 ; \mathrm{p}<0.01)$ but not in the primary prevention without modifiable risk factor group (1.01; 0.86-1.18; $\mathrm{p}=0.92)$

Conclusions: Statin treatment was not associated with a reduction in acute coronary syndrome or all-cause death in elderly without modifiable cardiovascular risk factor treated in primary prevention. 


\section{Introduction}

While there is clear consensus on the benefits of initiating statins in elderly patients with cardiovascular disease, the benefits of starting these treatments in elderly patients without such history remains debated given the lack of experimental or observational data focusing on primary prevention in the elderly, and the costs and risks of treatment. ${ }^{1-4}$ Moreover, the estimation of the cardiovascular risk (used to guide initiation of statins) may be overestimated in older adults by the usual tools that all consider age. ${ }^{5}$ Actually, the relevancy of the correlation established between cholesterol concentration and cardiovascular risk for younger adults is questioned when regarding the elders. ${ }^{6}$ In patients aged 80 years and over, a review of observational studies and clinical trials found that cholesterol was not predictive of mortality. ${ }^{7}$ Conversely, this review showed a trend towards increased mortality with low total cholesterol $(<5.5 \mathrm{mmol} / \mathrm{l})$ in this population. Despite this, statins are widely used in the elderly for primary prevention. ${ }^{8}$

In this context, we evaluated the real-life effectiveness of initiating statins among elderly patients, based on their cardiovascular risk.

\section{Methods}

\section{Setting}

This cohort study used data from the Echantillon Généraliste de Bénéficiaires (EGB) database. EGB is a $1 / 97^{\text {th }}$ representative sample of the population covered by the French national healthcare system (Système National des Données de Santé, SNDS), extensively used for pharmacoepidemiology research. ${ }^{9}$ It contains individual anonymous information on patients such as sex, year of birth, date of death (if applicable), all outpatient reimbursed healthcare expenditure, and information for a list of 30 specifically individualized chronic diseases for which the patient will receive full health expenses coverage. SNDS also includes 
the national hospital database covering both public and private hospitalizations, with information on dates of hospital stay, diagnoses (coded with International Classification of Diseases $10^{\text {th }}$ revision, ICD-10) and medical procedures. In accordance with regulations in place at the time of the study, the National Institute of Health and Medical Research (INSERM) was informed of the study that was to be performed using the EGB database.

There was no further requirement for ethical approval, or data protection agency approval for this study, which was done in fully anonymized data.

\section{Study population}

Patients were eligible for the study if they were aged 75 years and above during the study inclusion period (2009-2012) and affiliated to the general scheme of the French health insurance system (covering $78 \%$ of the population at the time). Every trimester between January $1^{\text {st }} 2009$ and December $31^{\text {st }} 2012$, new users of statin were dynamically included in the cohort. New users of statin were defined as a first identified statin dispensing without statin dispensing in the previous year.

To each statin new-user, one statin non-user was matched on age (+/- 2 years), gender, numbers of different drugs dispensed and of medical consultations in the previous 6 months, dispensing in the previous 6 months of cardiovascular drugs (antihypertensive drugs (diuretics, beta-blockers, calcium channel blockers (CCB), drugs acting on renin-angiotensinaldosterone system or other antihypertensive drugs), antiplatelet agents, and anticoagulants), and history of cardiovascular diseases (acute coronary syndrome, ischemic stroke, transient ischemic attack, and diabetes; see eTable 1 in the Supplement for identification codes).

Matched patients for whom one presented an outcome of interest (acute coronary syndrome or all-cause death) during the first month after index date (date of statin initiation for statin newusers or corresponding matching date for statin non-users) were not included, early outcome 
being considered as depending essentially on the medical history of the patient and only marginally on the effectiveness of statin initiation.

Patients were then categorized into three groups according to their cardiovascular risk at the time of the index date. Secondary prevention patients were patients with history of previous coronary heart disease (hospitalization in the year before index date or information of coronary heart disease as a specific ongoing chronic disease with full health expenses coverage). Primary prevention patients with modifiable cardiovascular risk factors were patients without history of previous coronary heart disease but with at least one of the following: diabetes, dispensing of antihypertensive drugs, antiplatelet agents or anticoagulants. Primary prevention patients without modifiable cardiovascular risk factor included all patients not included in the first two groups. Due to the nature of the healthcare claims database, modifiable risk factors such as smoking, overweight, physical activity or cholesterol levels cannot be identified unless patients benefit from a specific medical / drug treatment.

\section{Drug exposure}

Exposure to statins was estimated using dispensing data. The number of days covered for treatment by each dispensing was defined as the number of tablets dispensed (assuming a treatment schedule of one tablet per day) plus a grace period equal to $10 \%$ of the number of tablets dispensed. Owing to the lack of data for drug exposure during hospitalization, all periods of hospital stay were considered as periods of exposure if the patient was in possession of drugs at the date of hospital admission. ${ }^{10}$ For each month of follow-up, the periods of treatment coverage thus identified were used to determine whether the patient had received statin treatment during the month. Given the delayed clinical cardiovascular benefits of statins, the exposure to statins was defined as a time-dependent cumulative variable 
considering the total number of months on the drug, and expressed in terms of cumulated months of use.

\section{Outcomes}

The outcome of interest was the occurrence of acute coronary syndrome or all-cause death. Acute coronary syndrome was identified during follow-up as first hospitalization with ICD-10 diagnosis codes for unstable angina (I20.0), acute myocardial infarction (I21) or other acute ischemic heart diseases (I24). ${ }^{11}$

\section{Statistical analyses}

Patients were followed from their index date until their date of outcome (if appropriate), exit date (i.e. change in health insurance regimen, if appropriate), or until 31 December 2015, whichever happened first. Effect of cumulative use of statin was analyzed by multivariable time-dependent Cox models stratified on cardiovascular risk at inclusion (all covariates used in these models were listed in eTable 1 in the Supplement).

In the primary analysis, in addition to matching at inclusion on cardiovascular characteristics, models were adjusted for co-morbidities or co-medications considered as potential confounders and included as time-dependent variables (see eTable 1 in the Supplement). Moreover, owing to the potential impact of the presence of unmeasured confounders (database does not include information on lifestyle, lab values, and other potential confounders such as body mass index) models were also adjusted on deciles of a high-dimensional propensity score (hdPS) of statin initiation (see eMethod 1 in the Supplement). ${ }^{12-14}$

Multiple sensitivity analyses were performed to understand how confounding by healthy user or healthy adherer effects was handled by adjustment. ${ }^{15}$ First, given the 
persistence and adherence issues with statins, we considered a sensitivity definition of cumulative exposure to statin considering both the cumulative use and the cumulative non-use of the drugs. ${ }^{16}$ Under this sensitivity definition, the cumulative exposure to statin corresponded, at each month of follow-up, to the sum of cumulated months in possession of the drugs minus the number of cumulated months without the drugs since the beginning of follow-up (see eFigure 1 in the Supplement). Second, exposure to influenza vaccine, which is, in France, recommended and free of charge for all subjects aged 65 years or more, was used as a proxy of "healthy user" in the model adjustment. Third, new-users of CCB were considered as the reference group for the constitution of the study population instead of statin non-users in order to avoid healthy user bias. As described previously, this antihypertensive drug class could be considered as a negative drug control in this context given the absence of documented post-myocardial infarction survival advantages. ${ }^{17,18}$ Thereby, it could reflect adherence behavior of patients without influencing occurrence of outcome of interest. Finally, a sensitivity analysis was performed using only all-cause death as outcome.

All statistical analyses were performed using SAS® software (version 9.4, SAS Institute, NC, USA) and hdPS were estimated using the SAS Pharmacoepi Toolbox (version 2.4.15). ${ }^{19}$ Associations were estimated using hazard ratios (HR) and their $95 \%$ confidence intervals $(95 \% \mathrm{CI})$.

\section{Results}

\section{Patient characteristics}

Of the 5436 elderly patients who initiated statins between 2009 and 2012, $3642(67.0 \%)$ were matched 1:1 with statin non-users, for a total of 7284 patients included in this cohort (Figure 1). Over a median follow-up duration of 4.7 years, 2265 patients $(31.1 \%)$ had an acute coronary syndrome or died. 
Median age at inclusion was 80 years (IQR: 77-84); 2678 patients (36.8\%) were male; history of acute coronary syndrome was found in 346 patients (4.8\%), diabetes in 1772 patients (24.3\%), and dispensing of antihypertensive drugs in 5934 (81.5\%). Most patients were categorized in the group primary prevention with modifiable cardiovascular risk factors (84.9\%; Table 1). Compared to the 5436 statin new-users before matching, the 3642 statin new-users included in the cohort were less likely to be men (36.8\% vs. $40.2 \%)$, and had less frequent history of acute coronary syndrome $(4.8 \%$ vs. $9.8 \%)$ or ischemic stroke $(3.0 \%$ vs. $6.2 \%$ ) before index date (see eTable 2 in the Supplement).

Patients in primary prevention without modifiable cardiovascular risk factor were younger compared to those in primary prevention with modifiable cardiovascular risk factors and those in secondary prevention (median age: 78 years vs. 80 years and 83 years). They also had fewer co-morbidities: cardiac arrhythmia (4.1\% vs. $19.5 \%$ and $47.7 \%)$, chronic obstructive pulmonary disease (0.8\% vs. $3.0 \%$ and $11.0 \%)$, dementia $(5.3 \%$ vs. $7.3 \%$ and $10.4 \%)$, or heart failure (0.3\% vs. $8.2 \%$ and $37.3 \%)$. All patient baseline characteristics are detailed in Table 2 according to cardiovascular risk group. Among statin new-users, initiated statin treatments showed with lower intensity (drug or dosage) in patients in primary prevention without modifiable cardiovascular risk factor compared to patients in primary prevention with modifiable cardiovascular risk factors or patients in secondary prevention (see eTable 3 in the Supplement).

\section{Statin exposure}

Among statin new-users, exposure to statin represented $50.6 \%$ of follow-up time in the primary prevention without modifiable cardiovascular risk factor group, $62.8 \%$ in the primary prevention with modifiable cardiovascular risk factors group and, $72.5 \%$ in the secondary 
prevention group. A total of $15.4 \%$ of statin non-users at index date started statin therapy later during follow-up.

\section{Effectiveness of statins}

In the primary analysis, regardless of adjustment and the method used for adjustment, cumulative exposure to statin was not associated with a lower risk of acute coronary syndrome occurrence or all-cause death in primary prevention patients without modifiable cardiovascular risk factor (HR adjusted on time-dependent confounders and hdPS for statin initiation, highly adjusted: 1.01 per year of use, $95 \%$ CI 0.86-1.18, $\mathrm{p}=0.92$; Table 3). Conversely, cumulative exposure to statin was associated with a lower risk of events in primary prevention patients with modifiable cardiovascular risk factors (highly adjusted HR: 0.93 per year of use, $95 \%$ CI $0.89-0.96, \mathrm{p}<0.01$ ) and in secondary prevention patients (highly adjusted HR: 0.75 per year of use, $95 \%$ CI $0.63-0.90, \mathrm{p}<0.01)$. These results were comparable in the classically adjusted model (Table 3).

\section{Sensitivity analyses}

Sensitivity analyses considering both cumulative use and cumulative non-use of statin, and those considering adjustment for influenza vaccine (a proxy for "healthy user") showed results similar to primary analyses (see eTables 4 and 5 in the Supplement). Sensitivity analyses comparing new-users of statins to new-users of CCB did not impact results of statin effectiveness for patients in primary prevention with or without modifiable cardiovascular risk factors (see eTable 6 in the Supplement). However, as only 8 new-users of CCB in secondary prevention were included in the study population, HR could not be estimated in this group (see eTable 7 in the Supplement). Lastly, regardless of the CV risk, results of the sensitivity analyses using only all-cause death as outcome were similar to those using the 
combined outcome (see eTable 8 in the Supplement). Results of the sensitivity analyses are summarized in Figure 2.

\section{Discussion}

In patients aged 75 years and above, the effectiveness of statins was related to baseline cardiovascular risk. Regardless of treatment duration, initiating statins was not associated with lower risk of acute coronary syndrome or all-cause death in elderly patients without modifiable cardiovascular risk factor treated in primary prevention. Conversely, statin treatment was associated with a reduction in these events for patients in primary prevention with modifiable cardiovascular risk factors or in secondary prevention. The results were consistent across definitions of cumulative statin use including or not time without treatment to account for lack of adherence. They were also consistent regardless of adjustment method. Clinical trials evaluating statins efficacy that focus on the elderly are rare and to our knowledge the PROSPER and SAGE studies are the only ones specifically designed for this purpose. $^{20-22}$ SAGE considered only secondary prevention, and found reduced all-cause mortality in the elderly receiving intensive treatment with atorvastatin compared to moderate treatment with pravastatin. ${ }^{22}$ In PROSPER, after stratification on previous vascular disease at inclusion, benefit of pravastatin was found only in elderly patients with previous cardiovascular disease, who would correspond to the patients we defined as in secondary prevention. ${ }^{21}$ Finally in patients aged 65 years or more treated with statins in primary prevention, a meta-analysis found a reduction of the risk of myocardial infarction but no reduction of all-cause death. ${ }^{23}$ In the recently published re-analysis of the ALLHAT-LLT trial focusing on pravastatin in primary prevention, ${ }^{24}$ that included patients aged 65 years or more, there was actually reduction neither of cardiovascular events nor of all-cause death. Therefore, all of these RCTs results are in line with our nationwide observational analysis 
suggesting an effect of statins in patients at identified cardiovascular risk but not for patients without modifiable cardiovascular risk. This reinforces the need to consider cardiovascular risk estimation for the decision to treat patients with statin, and would plead to not pay excessive attention to isolated hypercholesterolemia, especially in the older population in which correlation between cholesterol level and cardiovascular mortality is poor. ${ }^{6,7}$

This study has several strengths, mostly related to the database used. The French Health Insurance system database is a nationwide database including comprehensive information for all out-of-hospital reimbursed health expenses and linked to the national database for hospitalization, it-self including comprehensive information on hospital stays. The source population considered for the analysis (i.e. affiliates to the National Health system General Scheme, for which follow-up is complete over the study period) is representative of more than three-quarters of the French population. ${ }^{9}$ For these affiliates, the database includes all reimbursed outpatient drugs and hospitalization events. Aside from patients not taking the dispensed drug, which is a limitation common to studies performed from claims database, the main potential misclassification for exposure is related to in-hospital intake (not informed in the database), the reason for which patients were considered as exposed during hospital stays to the drugs they were in possession of at the date of hospital admission. ${ }^{10}$ The main risk for misclassification of events, aside of coding errors in diagnosis, would relate to fatal and ignored acute coronary syndrome. However these events would be considered in the composite outcome which includes all-cause death, and all deaths are recorded in the database. Misclassifications relating to coding errors should also be minimal as we used a validated method to identify acute coronary syndrome (predictive positive value of $84.2 \%$; $95 \% \mathrm{CI},[72.1 ; 92.5])^{11}$

The present study nevertheless has certain limitations. The database does not include certain risk factors or potential confounders such as smoking, overweight, physical activity or 
cholesterol levels, which could result in an indication bias or healthy user bias. However, it does include proxies such as chronic obstructive pulmonary disease or diabetes for smoking and body mass index, respectively, which are strongly associated with the occurrence of cardiovascular events in patients with history of myocardial infarction. ${ }^{25}$ It also includes lowdose aspirin which is prescribed, reimbursed and recorded, and is also a strong predictor of coronary heart disease. However, despite this is considered to constitute one of the best markers of cardiovascular risk, it is possible that some aspirin low-dose elderly users were actually healthy patients taking aspirin prophylactically. Even this cannot be quantified, the amount of such is likely to be limited. The potential effect of unmeasured confounding should have been minimized in our study by adjustment on hdPS at inclusion associated to timedependent adjustment for multiple co-morbidities and co-medications. ${ }^{12-14}$ In addition, sensitivity analyses using CCB or influenza vaccine also confirmed the weak impact of healthy user bias on the results. Selection bias could have occurred; if so, it should have concerned essentially patients in the secondary prevention group, who appeared less represented and for whom the sex-ratio was slightly different in the population after matching. This might be explained by the fact that most patients with acute coronary syndrome receive secondary coronary prevention including statins in France, which makes it difficult to find unexposed controls to match with statin users among patients with acute coronary syndrome. The fact that women tend to be less intensively treated than men after an acute coronary syndrome could account for the sex-ratio shift. ${ }^{26,27}$ Despite this potential bias, the effect size herein reported was consistent with that estimated in meta-analyses of clinical trials in secondary prevention. ${ }^{28-30}$ The sensitivity definition we used for cumulative use of statin weighted equally a month of use and a month without treatment. By accounting for patients' lack of adherence or default in treatment persistence, this definition thus relies on a strong assumption that statin non-use had delayed clinical effects as well as that statin use had 
delayed benefits, the reason for which it was only retained for sensitivity purpose. Finally, as elderly patients in primary prevention without modifiable risk factors almost exclusively received low intensity treatment, we cannot presume that the lack of demonstration of the effectiveness of statin in this population would also worth for high intensity treatment. However, no clinical trial has demonstrated the efficacy of such intensive statin treatment for cardiovascular primary prevention in elderly patients yet.

Taken together, these results reinforce the idea of a rational use of statins in the older population. In elderly without significant cardiovascular risks, the benefits of statin treatment is low and might not outweigh side effects associated to the treatment. Moreover, its related cost is important for society. ${ }^{31}$ In our sample, $10 \%$ of statin initiators after the age of 75 were in primary prevention without modifiable cardiovascular risk factor, which would correspond to around 13000 patients yearly in France for which treatment benefit is questionable. Aside from considering not starting the drug, further research would however be needed to confirm that stopping statins is risk-free in these patients. ${ }^{32}$ Today, this was only pointed out in the context of life-limiting illness. ${ }^{33}$ 


\section{References}

1. Force USPST, Bibbins-Domingo K, Grossman DC, et al. Statin Use for the Primary Prevention of Cardiovascular Disease in Adults: US Preventive Services Task Force Recommendation Statement. Jama. Nov 15 2016;316(19):1997-2007.

2. Catapano AL, Graham I, De Backer G, et al. 2016 ESC/EAS Guidelines for the Management of Dyslipidaemias. European heart journal. Oct 14 2016;37(39):29993058.

3. Gurwitz JH, Go AS, Fortmann SP. Statins for Primary Prevention in Older Adults: Uncertainty and the Need for More Evidence. Jama. Nov 15 2016;316(19):1971-1972.

4. Godlee F. Lessons from the controversy over statins. Lancet. Mar 18 2017;389(10074):1100-1101.

5. Cooney MT, Selmer R, Lindman A, et al. Cardiovascular risk estimation in older persons: SCORE O.P. European journal of preventive cardiology. Jul 2016;23(10):1093-1103.

6. Krumholz HM, Seeman TE, Merrill SS, et al. Lack of association between cholesterol and coronary heart disease mortality and morbidity and all-cause mortality in persons older than 70 years. Jama. Nov 2 1994;272(17):1335-1340.

7. Petersen LK, Christensen K, Kragstrup J. Lipid-lowering treatment to the end? A review of observational studies and RCTs on cholesterol and mortality in 80+-year olds. Age and ageing. Nov 2010;39(6):674-680.

8. O'Keeffe AG, Nazareth I, Petersen I. Time trends in the prescription of statins for the primary prevention of cardiovascular disease in the United Kingdom: a cohort study using The Health Improvement Network primary care data. Clinical epidemiology. 2016;8:123-132. 
9. Bezin J, Duong M, Lassalle R, et al. The national healthcare system claims databases in France, SNIIRAM and EGB: Powerful tools for pharmacoepidemiology. Pharmacoepidemiol Drug Saf. Aug 2017;26(8):954-962.

10. Suissa S. Immeasurable time bias in observational studies of drug effects on mortality. American journal of epidemiology. Aug 1 2008;168(3):329-335.

11. Bezin J, Girodet PO, Rambelomanana S, et al. Choice of ICD-10 codes for the identification of acute coronary syndrome in the French hospitalization database. Fundamental \& clinical pharmacology. Dec 2015;29(6):586-591.

12. Rassen JA, Glynn RJ, Brookhart MA, Schneeweiss S. Covariate selection in highdimensional propensity score analyses of treatment effects in small samples. American journal of epidemiology. Jun 15 2011;173(12):1404-1413.

13. Schneeweiss S, Rassen JA, Glynn RJ, Avorn J, Mogun H, Brookhart MA. Highdimensional propensity score adjustment in studies of treatment effects using health care claims data. Epidemiology. Jul 2009;20(4):512-522.

14. Toh S, Garcia Rodriguez LA, Hernan MA. Confounding adjustment via a semiautomated high-dimensional propensity score algorithm: an application to electronic medical records. Pharmacoepidemiol Drug Saf. Aug 2011;20(8):849-857.

15. Shrank WH, Patrick AR, Brookhart MA. Healthy user and related biases in observational studies of preventive interventions: a primer for physicians. J Gen Intern Med. May 2011;26(5):546-550.

16. Bezin J, Groenwold RH, Ali MS, et al. Comparative effectiveness of recommended versus less intensive drug combinations in secondary prevention of acute coronary syndrome. Pharmacoepidemiol Drug Saf. Mar 2017;26(3):285-293.

17. Bertolet BD. Calcium antagonists in the post-myocardial infarction setting. Drugs Aging. Dec 1999;15(6):461-470. 
18. Rasmussen JN, Chong A, Alter DA. Relationship between adherence to evidencebased pharmacotherapy and long-term mortality after acute myocardial infarction. Jama. Jan 10 2007;297(2):177-186.

19. Rassen JA, Doherty M, Huang W, Schneeweiss S. Pharmacoepidemiology Toolbox. Boston, MA. http://www.hdpharmacoepi.org. Accessed 10 March 2016.

20. Lloyd SM, Stott DJ, de Craen AJ, et al. Long-term effects of statin treatment in elderly people: extended follow-up of the PROspective Study of Pravastatin in the Elderly at Risk (PROSPER). PloS one. 2013;8(9):e72642.

21. Shepherd J, Blauw GJ, Murphy MB, et al. Pravastatin in elderly individuals at risk of vascular disease (PROSPER): a randomised controlled trial. Lancet. Nov 23 2002;360(9346):1623-1630.

22. Deedwania P, Stone PH, Bairey Merz CN, et al. Effects of intensive versus moderate lipid-lowering therapy on myocardial ischemia in older patients with coronary heart disease: results of the Study Assessing Goals in the Elderly (SAGE). Circulation. Feb 13 2007;115(6):700-707.

23. Savarese G, Gotto AM, Jr., Paolillo S, et al. Benefits of statins in elderly subjects without established cardiovascular disease: a meta-analysis. Journal of the American College of Cardiology. Dec 3 2013;62(22):2090-2099.

24. Han BH, Sutin D, Williamson JD, et al. Effect of Statin Treatment vs Usual Care on Primary Cardiovascular Prevention Among Older Adults: The ALLHAT-LLT Randomized Clinical Trial. JAMA Intern Med. Jul 1 2017;177(7):955-965.

25. Blin P, Dureau-Pournin C, Lassalle R, et al. Outcomes in patients after myocardial infarction similar to those of the PEGASUS-TIMI 54 trial: A cohort study in the French national claims database. Br J Clin Pharmacol. Sep 2017;83(9):2056-2065. 
26. Virani SS, Woodard LD, Ramsey DJ, et al. Gender disparities in evidence-based statin therapy in patients with cardiovascular disease. The American journal of cardiology. Jan 1 2015;115(1):21-26.

27. Salvo F, Bezin J, Bosco-Levy P, et al. Pharmacological treatments of cardiovascular diseases: Evidence from real-life studies. Pharmacol Res. Apr 2017;118:43-52.

28. Baigent C, Keech A, Kearney PM, et al. Efficacy and safety of cholesterol-lowering treatment: prospective meta-analysis of data from 90,056 participants in 14 randomised trials of statins. Lancet. Oct 8 2005;366(9493):1267-1278.

29. Cannon CP, Steinberg BA, Murphy SA, Mega JL, Braunwald E. Meta-analysis of cardiovascular outcomes trials comparing intensive versus moderate statin therapy. Journal of the American College of Cardiology. Aug 1 2006;48(3):438-445.

30. Cholesterol Treatment Trialists C, Fulcher J, O'Connell R, et al. Efficacy and safety of LDL-lowering therapy among men and women: meta-analysis of individual data from 174,000 participants in 27 randomised trials. Lancet. Apr 11 2015;385(9976):13971405

31. Curfman G. Risks of Statin Therapy in Older Adults. JAMA Intern Med. Jul 1 2017;177(7):966.

32. Bonnet F, Poulizac P, Joseph JP. Safety and efficacy of statins. Lancet. Mar 18 2017;389(10074):1097-1098.

33. Kutner JS, Blatchford PJ, Taylor DH, Jr., et al. Safety and benefit of discontinuing statin therapy in the setting of advanced, life-limiting illness: a randomized clinical trial. JAMA Intern Med. May 2015;175(5):691-700. 


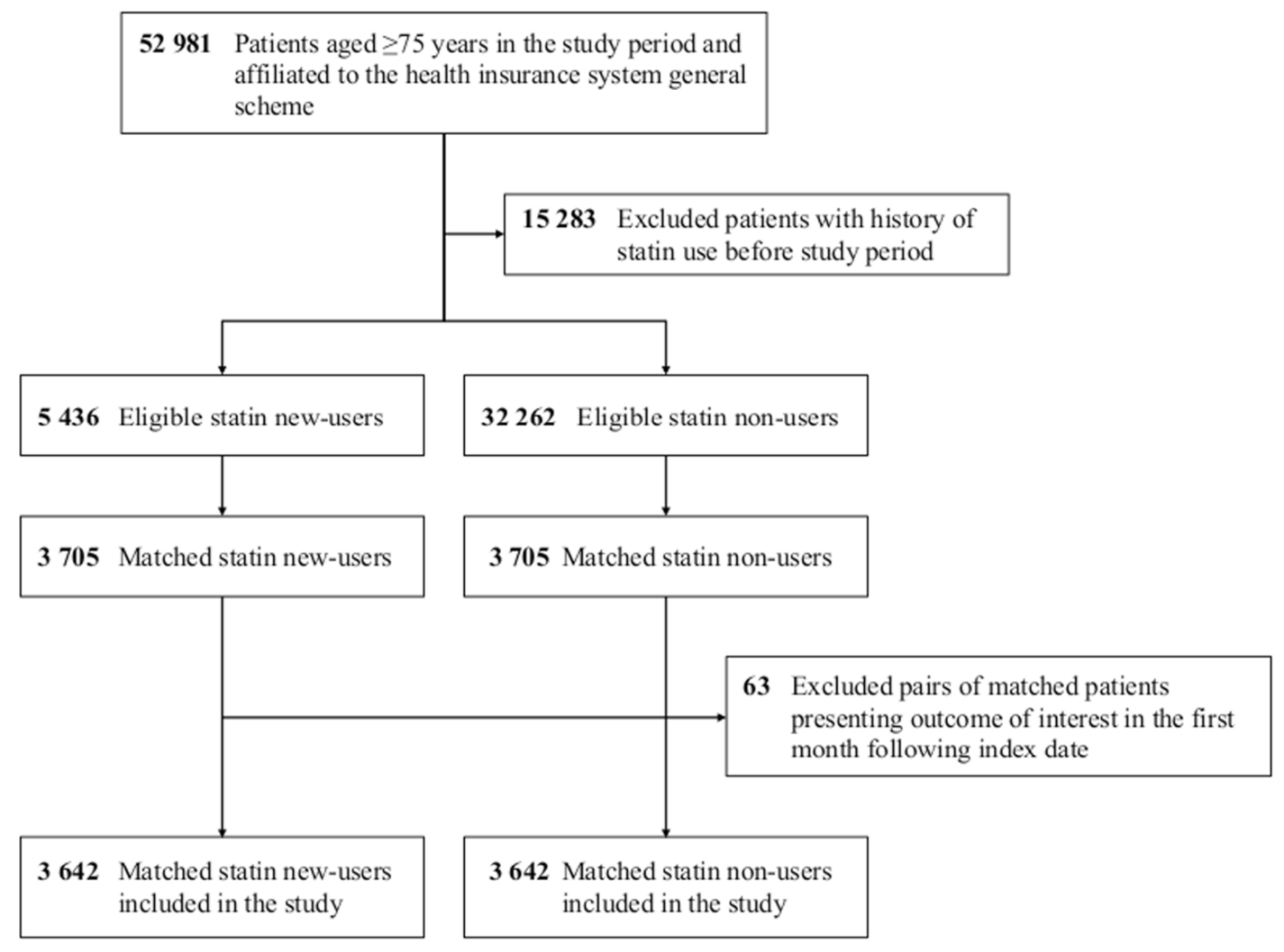

Figure 1. Study flow chart. 
Person-years (N) Events (N) Hazard Ratio, 95\% Cl

Primary prevention without modifiable risk factor Primary analysis

Sensitivity analyses

Sensitivity definition for cumulative exposure

Adjustement for exposure to influenza vaccine

New-users of CCB as negative group control

Only all-cause death as outcome

Primary prevention with modifiable risk factors

Primary analysis

Sensitivity analyses

Sensitivity definition for cumulative exposure

Adjustement for exposure to influenza vaccine

New-users of $\mathrm{CCB}$ as negative group control

Only all-cause death as outcome

\section{Secondary prevention}

Primary analysis

Sensitivity analyses

Sensitivity definition for cumulative exposure

Adjustement for exposure to influenza vaccine

New-users of CCB as negative group control

Only all-cause death as outcome
3728

3728

3728

1971

3784

$\begin{array}{llll}27442 & 1948 & 0.93 & (0.89-0.96) \\ 27442 & 1948 & 0.93 & (0.89-0.97) \\ 27442 & 1948 & 0.93 & (0.90-0.97) \\ 9565 & 619 & 0.91 & (0.84-0.98) \\ 28106 & 1770 & 0.92 & (0.88-0.95)\end{array}$

$1094 \quad 201 \quad 0.75(0.63-0.90)$

$1094 \quad 201 \quad 0.74(0.61-0.89)$

$1094 \quad 201 \quad 0.74(0.61-0.90)$

42

1276

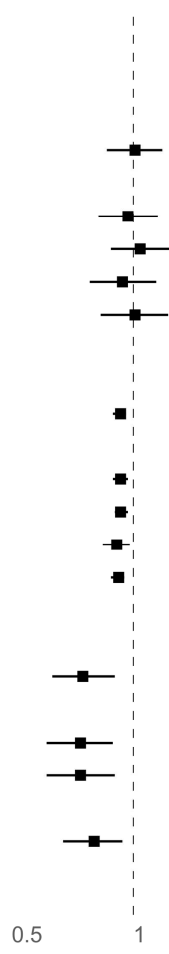

Figure 2. Sensitivity analyses adjusted hazard ratio point estimates and their $95 \%$ confidence intervals (95\%CI). Adjustment performed on time-dependent confounders and high dimensional propensity score for statin initiation. Abbreviation: CCB, Calcium Channel Blocker. 
Table 1. Description of matching variables at inclusion according to statin use

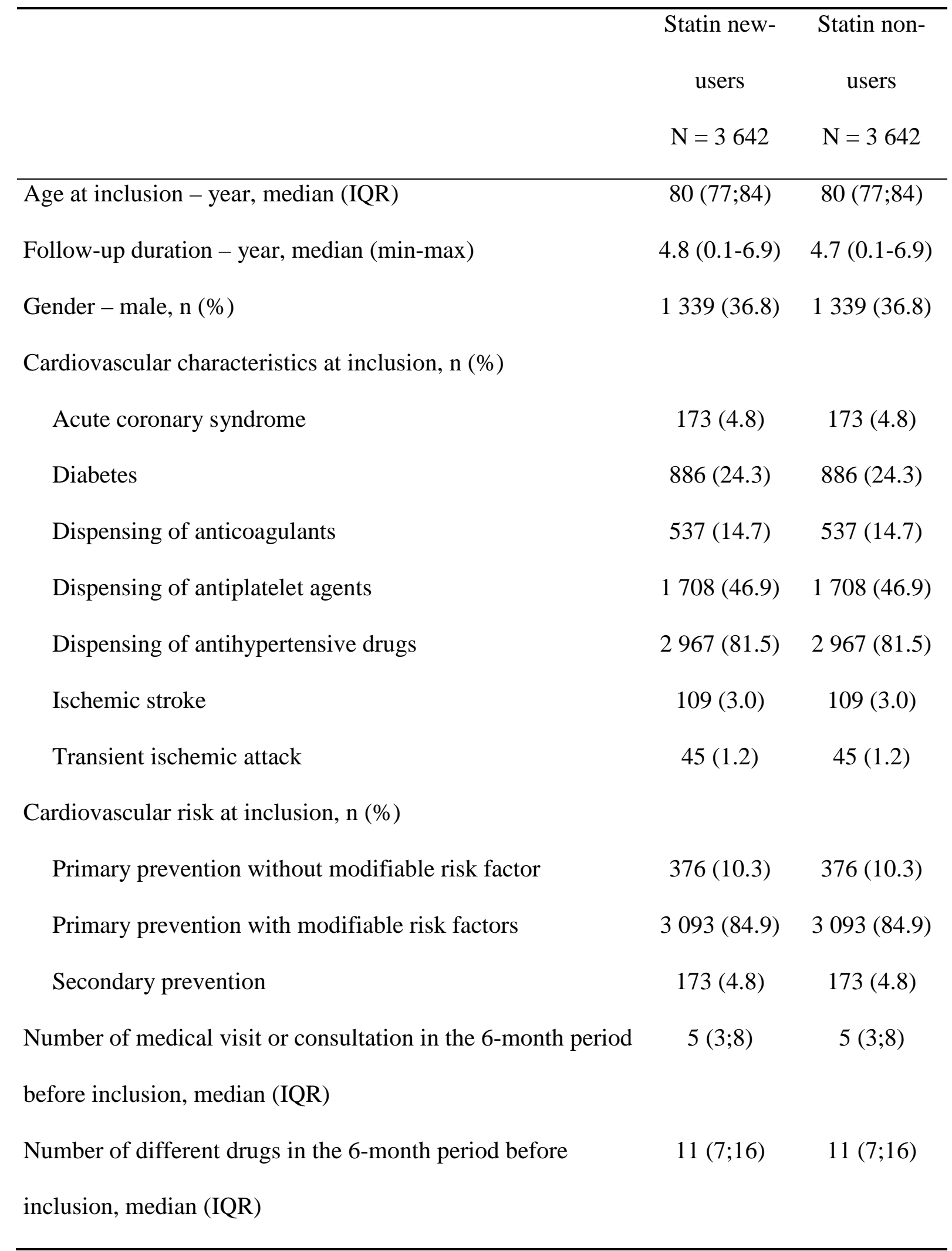

Abbreviation: IQR: interquartile range 
Table 2. Patient characteristics at inclusion according to cardiovascular risk

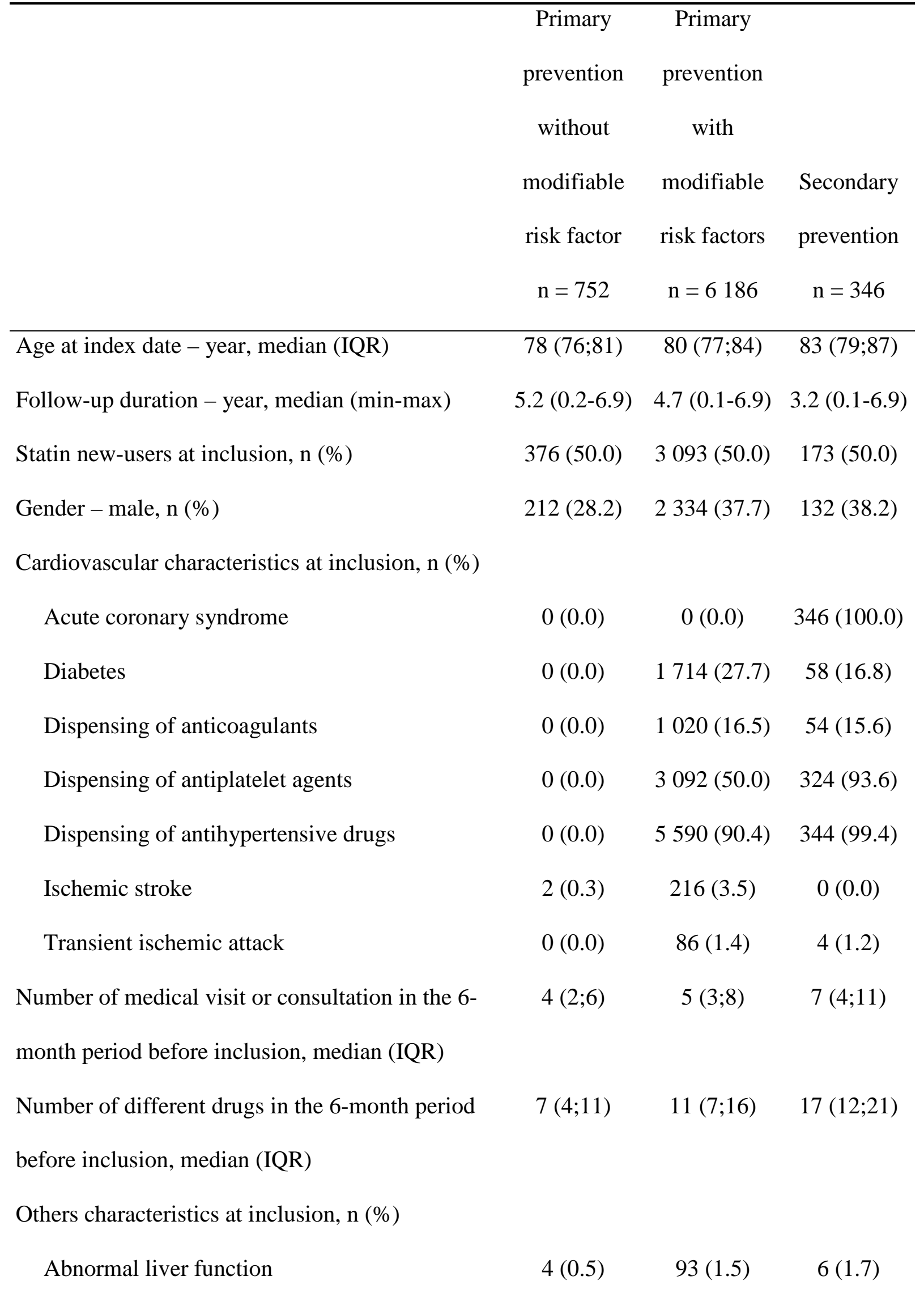




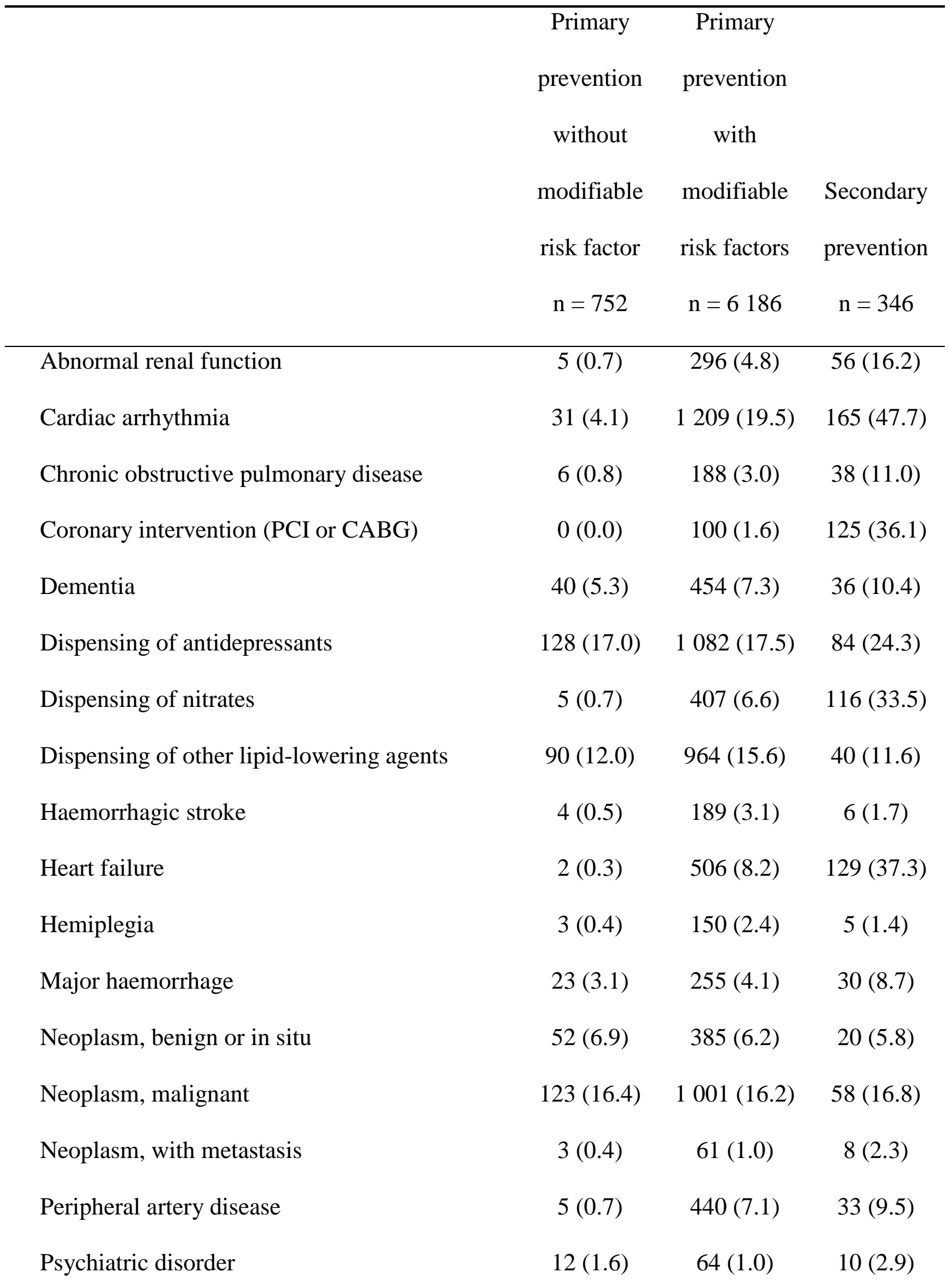

Outcome of interest during follow-up, n (\%) 


\begin{tabular}{lcccc}
\hline & Primary & Primary & \\
& prevention & prevention & \\
& without & with & \\
& modifiable & modifiable & Secondary \\
& risk factor & risk factors & prevention \\
& n $=752$ & $\mathrm{n}=6186$ & $\mathrm{n}=346$ \\
\hline All-cause death & $99(13.2)$ & $1770(28.6)$ & $171(49.4)$ \\
Acute coronary syndrome or all-cause death & $116(15.4)$ & $1948(31.5)$ & $201(58.1)$ \\
\hline
\end{tabular}

Abbreviations: $C A B G$ : coronary artery bypass grafting; IQR: interquartile range; PCI: percutaneous coronary intervention 


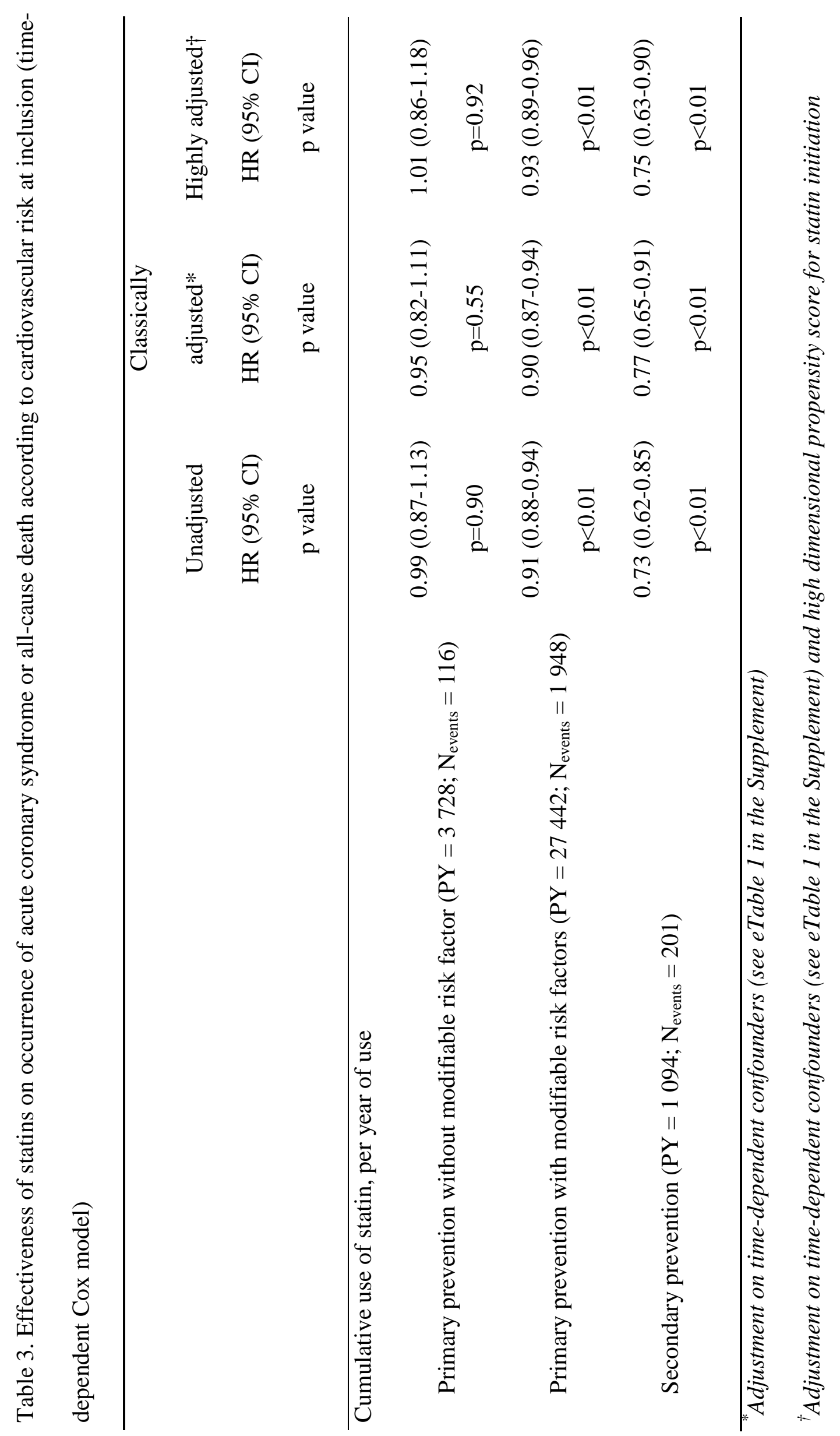


$\ddot{2}$

$\stackrel{2}{3}$

0
0
0
0

$\dot{8}$

$\frac{0}{5}$

产

$\frac{3}{3}$

ป⿱艹

$\frac{8}{8}$

ำ

$\frac{3}{3}$

苛

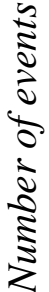

そั

$\ddot{\vec{\Xi}}$

ప

$\frac{0}{3}$

ن

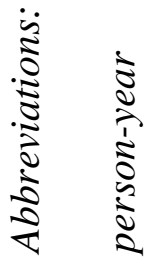

